\title{
BAG-1 Stabilizes Mutant F508del-CFTR in a Ubiquitin-Like-Domain-Dependent Manner
}

\author{
Filipa Mendes ${ }^{a, b, c} \quad$ Carlos M. Farinha ${ }^{b}$ Verónica Felício ${ }^{b} \quad$ Paulo C. Alves ${ }^{a}$ \\ Isabel Vieira ${ }^{a}$ Margarida D. Amarala ${ }^{a, b}$
}

aDepartment of Human Genetics, National Institute of Health, Lisboa; ${ }^{b}$ Center for Biodiversity, Functional and Integrative Genomics - Faculty of Sciences, University of Lisboa, Lisboa; 'Current address: Chemical and Radiopharmaceutical Sciences Unit, IST/ITN, Instituto Superior Técnico, Technical University of Lisboa, Sacavém

\author{
Key Words \\ Cystic Fibrosis • CFTR • ERAD • BAG-1 • Hsp70 • Ubiquitin
}

\begin{abstract}
Background: Cystic Fibrosis Transmembrane Conductance Regulator (CFTR), the dysfunctional $\mathrm{Cl}^{-}$channel in Cystic Fibrosis, undergoes complex biosynthesis at the endoplasmic reticulum involving several molecular chaperones including Hsp70 and many co-chaperones. $\mathrm{B} c \mathrm{l}-2-$ associated athanogenes (BAGs) constitute a protein family sharing an Hsc70-binding domain. BAG-1 possesses an ubiquitin-like domain (Ub-LD) responsible for proteasomal association and for promoting substrate release from $\mathrm{Hsc70/Hsp70}$ in vitro by accelerating the chaperone ATP/ADP exchange rate. Methods: Herein, we studied the in vivo effect of BAG-1 on the turnover and processing of wild type (wt)- and F508del-CFTR, the most frequent mutation in CF patients. Results: Results show that BAG-1 associates with both wt- and F508del-CFTR (in higher yields with the latter) through its Ub-LD and independently of Hsc70. Moreover, the immature form of F508del-CFTR (but not of wt-CFTR) is stabilized by BAG-1 overexpression, albeit in a cell-type specific way, without detectable maturation. Data also show that BAG-1 and the proteasome inhibitor ALLN are not additive on stabilizing F508del-CFTR and this effect depends on BAG-1 Ub-LD. Moreover, under BAG-1 overexpression, a reduction in ubiquitinylated-CFTR occurs suggesting that BAG-1 competes with Ub. Conclusion: Overall, data are compatible with a mechanism in which BAG-1 stabilizes F508del-CFTR by direct binding, probably competing out ubiquitin to partially avoid its proteasomal degradation.
\end{abstract}

Copyright @ 2012 S. Karger AG, Basel 


\section{Introduction}

The genetic disease Cystic Fibrosis is caused by mutations in the Cystic Fibrosis Transmembrane Conductance Regulator (CFTR) which lead to the absence or malfunction of CFTR protein, a cAMP-regulated $\mathrm{Cl}^{-}$channel localized at the apical membrane of epithelial cells. The most prevalent disease-causing mutation, found in about $90 \%$ of CF patients, is the deletion of a phenylalanine residue at position 508 (F508del) [1].

CFTR is a member of the ABC (ATP-binding cassette) transporters super-family, and its structure comprises two membrane-spanning domains (MSDs), two nucleotide-binding domains (NBDs) and a regulatory domain (RD). Given its multi-domain structure, CFTR undergoes a fairly complex biosynthetic pathway at the endoplasmic reticulum (ER) [2] in which several molecular chaperones and co-chaperones from both the ER-membrane and the cytosol have been shown to participate. These include: Hsp70/Hsc70 [3], calnexin[4, 5], Hdj-2 [6], Hsp90 [7], Hdj-1/Hdj-2 [5, 8], HspBP1 [9], (reviewed in [2]), CHIP, BAG-2 [10] and cysteine string protein (Csp) shown to regulate the exit of CFTR from the ER and Hsc70/ Hsp70-CHIP-mediated CFTR degradation [11].

CFTR biogenesis initiates with its co-translational folding into the ER membrane where the protein is core-glycosylated, originating an immature precursor form ( $145 \mathrm{KDa})$, known as band B. Wild-type (wt)-CFTR undergoes maturation through the Golgi, originating a $\sim 170-k D a$ complex form (band C) [12]. Depending on the cell type, only a fraction (25\% to $70 \%$ ) of precursor wt-CFTR matures [13], the remaining undergoes ubiquitination (Ub) and degradation by the proteasomal pathway (UPP) [14,15]. Far less F508del-CFTR (undetectable in most cell types) leaves the ER due to its inefficient folding and incomplete conformational maturation [13]. Indeed, the ER quality control (ERQC) targets the misfolded protein to UPPdegradation $[14,15]$ and thus very little protein reaches the cellular membrane. However, proteasome inhibitors only partially prevent CFTR degradation, indicating that key factors in the CFTR degradative pathway remain unidentified $[14,15]$.

We have previously shown that co-overexpressing Hsp70 and Hsp40 [5] stabilizes wtbut not F508del-CFTR. Our studies with glycosidase inhibitors and the ER-chaperone calnexin have also shown that calnexin-mediated and glycan-dependent ER-associated degradation (GERAD) occurs for wt-CFTR, but that most F508del-CFTR undergoes glycan-independent ER-associated degradation (ERAD) through an earlier, plausibly Hsc70/Hsp70-mediated proteolytic pathway [16]. These results, together with data from several other groups, led us to propose a multi-checkpoint model for the ERQC where wt- and F508del-CFTR are mostly discarded at two different major ERQC checkpoints, having as major mediators the calnexin and Hsc70-machineries, respectively [16]. More recently, we have proposed the existence of a third checkpoint mediated by recognition of arginine-framed tripeptides, or AFTs [17]. Notwithstanding, the ERQC checkpoint mediated by Hsc70 remains the most critical for retention and degradation of F508del-CFTR [16].

A number of Hsc70 interactors (e.g., co-chaperones) are known to regulate Hsc70 activity towards a pro-folding or pro-degradative pathway for Hsc70 client proteins (reviewed in [2]). Among these are the Bcl-2-associated athanogenes (BAGs)- family proteins, which were originally identified by their ability to associate with the anti-apoptotic protein Bcl-2 [18]. Thus far, BAG proteins have been reported to affect diverse cellular behaviours ranging from cell division and apoptosis to cell migration and differentiation by modulating the activity of molecular chaperones, protein kinases, receptor signalling and transcription factors [19]. BAG-1, the founding member of the BAG family $[18,20]$ is also a co-chaperone of Hsc70 [21, $22]$ and in vitro it was shown to promote substrate release from Hsc70 and ATP hydrolysis by accelerating the ATP/ADP exchange rate [21,22]. BAG-family proteins share a conserved domain of approximately 100 amino acids in length responsible for Hsc70 binding, also called the BAG domain (BD), which modulates (positively or negatively) the activity of this chaperone, but differ in their $\mathrm{N}$-terminus depending on the translation initiation site. [19]. BAG-1 also possesses an Ub-like domain (Ub-LD) at its amino-terminus which was proposed to constitute a physical link between the Hsp70/Hsc70 machinery and the proteasome 
[23]. Indeed, BAG-1 was proposed to influence Hsp70/Hsc70 decision between promoting folding or degradation of its substrates $[23,24]$. It was also put forward that both BAG-1 and BAG- 2 regulate the activity of the E3-Ub-ligase, CHIP: BAG-1 stimulating CHIP-mediated degradation, while BAG-2 inhibiting it [10]. Recently it has been shown in a reconstituted cell-free system, that the pro-folding role of Hsc70 during co-translational CFTR folding is counterbalanced by its role in posttranslational targeting to UPP. Importantly, this balance was regulated by the integrated expression of co-chaperones such as BAG-1 [25].

Here, we examined the in vivo effect of overexpressing murine BAG-1 on the biogenesis and maturation of wt- and F508del-CFTR and explore its in vivo interaction with CFTR and Hsc70, by using various BAG-1 mutants lacking the HSP70 binding domain and the Ub-LD. Murine BAG-1, which we chose to examine here, corresponds to the human BAG-S isoform of $26 \mathrm{kDa}[18,26]$. Our findings indicate that the immature form of F508del-CFTR (but not of wt-CFTR) is stabilized by BAG-1 overexpression in a Ub-LD-dependent manner, but without detectable maturation of F508del-CFTR. We find that BAG-1 can bind CFTR directly i.e., independently of its Hsp70-binding domain. Our data also indicate that stabilization results from competition between BAG-1 and Ub.

\section{Materials and Methods}

\section{Cell lines, Plasmids and Transfections}

Baby hamster kidney (BHK), chinese hamster ovary cells (CHO) cell lines stably transfected with wt- and F508del-CFTR and cystic fibrosis bronchial epithelial cells (CFBE) transduced with either wt- or F508del-CFTR were cultivated as previously described [27, 28]. CHO K1 (CCL61) were purchased from ATCC (Manassas, VA, USA). When indicated in the figure legend, cells were submitted to stress by heat shock (HS) for $90 \mathrm{~min}$ at $43^{\circ} \mathrm{C}$, and then allowed to recover at $37^{\circ} \mathrm{C}$ for various periods of time.

We used murine BAG-1 cDNA because it is the closest to hamster, which is organism of origin of the CFTR-stable expressing cells we used in the present study. Two isoforms of BAG-1 have been identified in mouse; BAG-1S (p32) and BAG-1L (p50) [29]. We used the murine BAG-1S (p32) cDNA (Accession no. NM-009736) encoding 222 aa which was excised from pGEX-4T-1 with an EcoRI/XhoI hydrolysis [18]. This fragment was cloned into pEGFP-C2 (Clontech, Mountain View, CA, USA) and pcDNA3 (Invitrogen, Paisley, UK). The following deletion mutants of murine BAG-1 were used: BAG-1- $\Delta \mathrm{N}$ missing the first 89 aa, i.e., lacking the Ub-like domain (Ub-LD), BAG-1- $\Delta$ C lacking the last 47 aa, and BAG-1 90-200, missing the first 90 aa (Ub-LD), and the last 19 aa, i.e., part of the Hsc70/Hsp70 interacting domain [30]. All these mutants were cloned into pcDNA3. Lipofectin (Invitrogen) was used in transient transfections to deliver $5 \mu \mathrm{g}$ of the constructs to $\mathrm{CHO}$ and BHK cells stably expressing wt- or F508del-CFTR grown in p60 dishes.

Expression of BAG-1 was down-regulated using specific siRNA (Silencer Select s1865 from Ambion). CFBE-wt or F508del cells seeded on 96-well plates were transfected with 1.2 pmol of siRNA (either specific to BAG-1 or negative control) and analysed at $48 \mathrm{~h}, 72 \mathrm{~h}$ or $96 \mathrm{~h}$ post-transfection.

\section{Antibodies}

The antibodies (Abs) used for CFTR were the mouse monoclonal Ab M3A7, (Chemicon, Temecula, CA, USA) or the rabbit polyclonal Ab 169 [31]. For BAG-1 the Abs used were the rabbit polyclonal PC115 (Calbiochem, San Diego, CA, USA) and the goat polyclonal AF815 (R\&D systems, Minneapolis, MN, USA), raised against human and mouse BAG-1, respectively and the rabbit polyclonal sc-939 (Santa Cruz Biotechnology, Santa Cruz, CA, USA) recommended for detection of Bag-1 from mouse, rat and human. Other antibodies were: rat monoclonal anti-Hsc70 SPA-815 and rabbit anti-Hsp70 SPA-812 from Stressgen (Victoria, BC, Canada), anti-beta actin AC-40 from Sigma-Aldrich (St Louis, MO, USA) and anti-Ubiquitin FK1 from Biomol (Plymouth Pike, PA, USA).

\section{SDS-PAGE and immunoblotting}

Cells were lysed with sample buffer [1.5\% (w/v) SDS, $5 \%(\mathrm{v} / \mathrm{v})$ glycerol, $0.001 \%(\mathrm{w} / \mathrm{v})$ Bromophenol Blue, $0.5 \mathrm{mM}$ dithiothreitol and $31.25 \mathrm{mM}$ Tris, $\mathrm{pH}$ 6.8]. Total protein was quantified by a modified micro Lowry method and aliquots were loaded onto $7 \%$ or $10 \%(\mathrm{w} / \mathrm{v})$ polyacrylamide gels for electrophoretic 
separation and transferred onto nitrocellulose filters (Schleicher \& Schuell, Dassel, Germany). The filters were probed with anti-BAG-1, anti-Hsp70 or anti-CFTR Abs (see above). As a loading control, blots were re-probed with the anti-beta-actin $\mathrm{Ab}$ (see above). Blots were developed using the SuperSignal West Pico Chemiluminescent system (Pierce, RockFord, IL, USA).

Pulse-chase and CFTR immunoprecipitation (IP)

Twenty-four hours post-transfection cells were starved for $30 \mathrm{~min}$ in methionine-free medium and then pulsed for $30 \mathrm{~min}$ at $37^{\circ} \mathrm{C}$ in the same medium supplemented with $150 \mu \mathrm{Ci}\left[{ }^{35} \mathrm{~S}\right]$-methionine (ICN Biomedicals, Irvine, CA, USA). Pulse-chase experiments, followed by IP with an anti-CFTR Ab were performed as previously described [5]. Where indicated, cells were incubated before the pulse with $200 \mu \mathrm{M}$ ALLN (Sigma) for $120 \mathrm{~min}$ [32]. Briefly, cells were chased for the indicated time periods in complete medium supplemented with $1 \mathrm{mM}$ nonradioactive methionine, lysed in $1 \mathrm{ml}$ of RIPA buffer and CFTR IP carried out with an anti-CFTR Ab and Protein G-Agarose beads (Roche, Basel, Switzerland). Immunoprecipitated proteins were eluted with sample buffer and then separated by SDS-PAGE. SDS-PAGE, fluorography, densitometry and statistical analysis were also as described [5]. Differences are considered as significant for $p$ values $<0.05$.

Pull-down assays

For the pull-down assays, cells were lysed in chaperone buffer [50 mM Tris, $150 \mathrm{mM} \mathrm{NaCl}, 10 \mathrm{mM}$ ammonium molybdate, $0.09 \%$ (v/v) NP40, pH 7.4] and CFTR was immunoprecipitated with an anti-CFTR $\mathrm{Ab}$ and Protein G-Agarose beads as above (Roche). Immunoprecipitated proteins were eluted with sample buffer for $1 \mathrm{~h}$ at room temperature, electrophoretically separated by SDS-PAGE and immunoblotted as described above.

\section{Results}

$B A G-1$ expression after transient transfection and under stress conditions

Since BAG-1 acts as a modulator of the activity of Hsc70/Hsp70 chaperone, we tested whether its overexpression affects biosynthesis or maturation of wt-CFTR and F508delCFTR. First, we transfected cells stably expressing the normal or mutant (F508del) forms of CFTR with a recombinant expression vector containing BAG-1 cDNA, or with the empty vector (mock) as a negative control and we evaluated the expression of the heterologous BAG1 (mouse) by Western blot (Fig. 1A). Results obtained show that the AF815 antibody (Ab) did not detected BAG-1 in mock-transfected cells (lanes 1,3) but only in significant levels $24 \mathrm{~h}$ post-transfection with the BAG-1 construct, either in wt- or F508del-CFTR CHO expressing cells (lanes 2,4, respectively). The BAG-1 Ab used here is able to detect the 3 different BAG-1 mouse isoforms, as described by the manufacturer (see Methods). Densitometric analysis of the results evidences the increase in the amount of immature F508del-CFTR at steady state under BAG-1 overexpression (Fig. 1A, lane 4, upper panel and Fig. 1B).

To look for a possible effect of BAG-1 in regulating Hsp70 expression (or vice-versa), which in turn might influence CFTR biogenesis [5, 33], we then assessed Hsp70 levels under heat-shock (HS) stress (Fig. 1C) in CHO cells with and without BAG-1 overexpression. By using the SPA-812 anti-Hsp70 Ab, which does not recognize the constitutive variant Hsc70 (see Methods), Hsp70 was only immunodetected when wt- or F508del-CFTR cells were subjected to HS (lanes 2, 4, 6 and 8). These results confirm a HS triggered Hsp70 induction as expected. However, neither BAG-1 nor the liposome transfection per se induced a stress response, as shown by lack of Hsp70 induction in either mock- (lanes 1,5) or BAG1-transfected non-HS cells (lanes 3, 7), in contrast to viral methods of DNA delivery [34]. Interestingly, the induction of Hsp70 which is very intense at recovery post-HS (18h) in mock-transfected cells (lanes 2 and 6 in Fig. 1C) as expected, is drastically reduced in wtor F508del-CFTR cells overexpressing BAG-1 (Fig. 1C, lanes 4 and 8). Conversely, BAG-1transfection consistently resulted in lower BAG-1 expression levels in cells subjected to HS 
A.

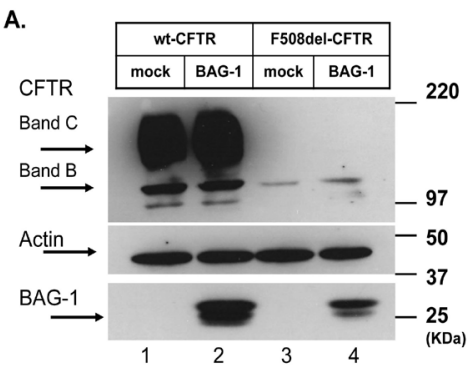

c.

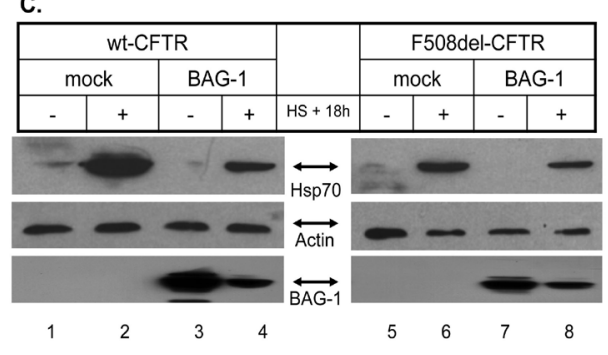

B.

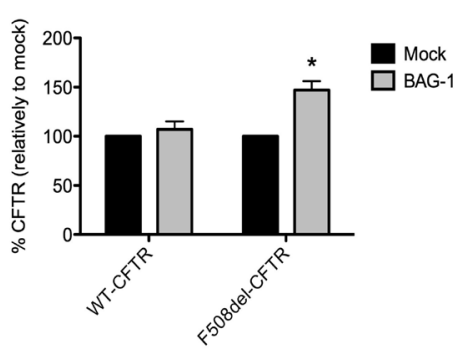

D.

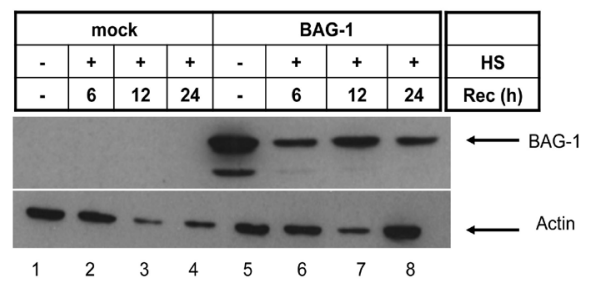

Fig. 1. Immunodetection of heterologously expressed BAG-1 (Bcl-2-associated athanogenes) and its effect on Hsp70 induction by heat-shock (HS). (A) BAG-1 was immunodetected with the AF815 antibody (Ab) in Chinese hamster ovary (CHO) cells stably expressing wild-type (wt)- or F508del-CFTR 24h after being transiently transfected with pcDNA3 murine BAG-1 construct (lanes 2 and 4) or with an empty pcDNA3 vector as a mock control (lanes 1 and 3). CFTR expression levels were also assessed in the same cells after BAG- 1 transfection and as a loading control, filters were probed for $\beta$-actin. (B) Densitometric analysis of Western blots showing the percentage of either wt- or F508del-CFTR at steady state in BAG-1 transfected cells normalized to the amount in mock-transfected cells. Asterisk indicates statistically significant difference from the mock-transfected cell line $(\mathrm{p}<0.05)$. (C) Hsp70 was immunodetected with SPA-812 Ab in CHO wtCFTR or F508del cells 24h post-transfection either with the BAG-1 pEGFP-C2 construct (lanes 3, 4, 7 and 8) or with the empty vector (lanes 1, 2, 5 and 6). To test whether BAG-1 affects induction of Hsp70 by HS, cells were analyzed either at 18h post-recovery from HS (lanes 2, 4, 6 and 8) or without stress (lanes 1, 3, 5 and 7). To assess for BAG-1 overexpression, filters were also probed for BAG-1. As a loading control, filters were probed for $\beta$-actin (lower panels). (D) BAG-1 was immunodetected with the AF815 Ab in CHO wt-CFTR cells $24 \mathrm{~h}$ after transient transfection with pEGFP-BAG-1 (lanes 5-8) or with empty pEGFP (lanes 1-4) at various times (8h, 12h, 24h) after recovery from a HS of $90 \mathrm{~min}$ at $43{ }^{\circ} \mathrm{C}$ (lanes $2-4$ and 6-8) or without HS (lanes 1 and 5). As a loading control, filters were probed for $\beta$-actin.

and 6h-, 12- or 24h-recovery (Fig. 1D, lanes 6, 7 and 8, respectively), in comparison to nonHS cells (Fig. 1D, lane 5).

\section{In vivo effect of BAG-1 on the processing and degradation of wt-CFTR}

To assess the effect of BAG-1 overexpression on the turnover rate and processing efficiency of wt-CFTR, metabolic pulse-chase experiments followed by CFTR IP were performed in CHO cells stably expressing wt-CFTR (Fig. 2A).

Data show no apparent effect of BAG-1 overexpression either in the turnover rate of immature wt-CFTR (band B) or in its processing efficiency into the mature form, band $C$ (Fig. 2A, lanes 7-12) in comparison to mock-transfected cells (Fig. 2A, lanes 1-6). Results plotted graphically after densitometric analysis of immature and mature forms validate these observations (Fig. 2B and C, respectively). Indeed, statistical comparison of the slopes of the two lines (see Methods) confirmed that the degradation rate of the core-glycosylated form of wt-CFTR (band B) after BAG-1 transfection was not significantly altered relative to mock-transfected cells (Fig. 2B). Similarly, no significant difference occurred between the 


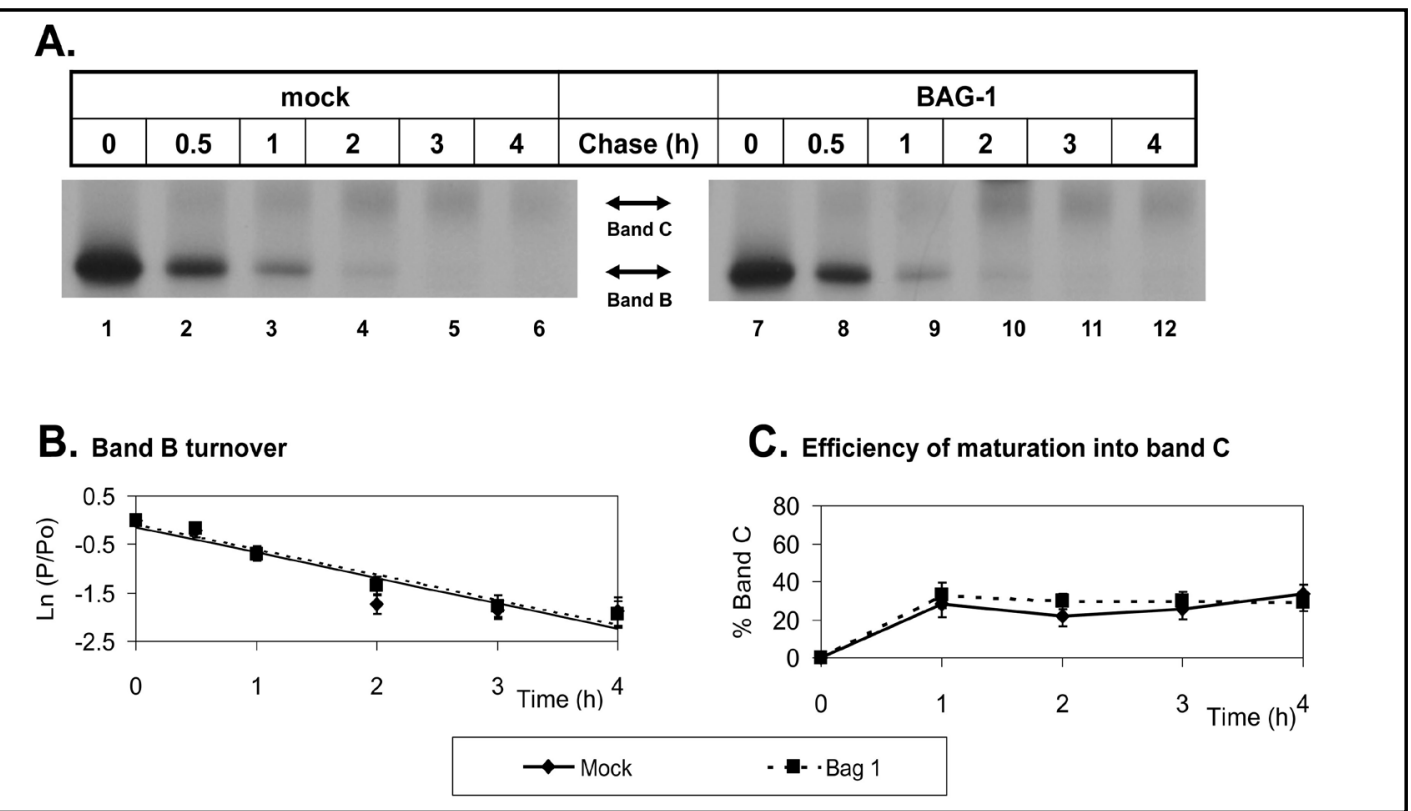

Fig. 2. BAG-1 overexpression does not affect turnover or processing of wt-CFTR. (A) CHO cells stably expressing wt-CFTR were transfected with the pcDNA3 BAG-1 construct (lanes 7-12) or with the empty pcDNA3 vector (lanes 1-6). 24h post-transfection, cells were subjected to a 30 min pulse-labelling with [ $\left.{ }^{35} \mathrm{~S}\right]$ methionine and chased for the indicated time periods. After cell lysis, CFTR was immunoprecipitated (IP) and separated by SDS-PAGE followed by fluorography (see Methods). Arrows indicate the position of bands $\mathrm{B}$ and $\mathrm{C}$ of CFTR. (B) Turnover rate of the immature form (band B) of wt-CFTR in CHO cells transfected with BAG-1 or mock-transfected. As the turnover of immature CFTR is exponential, results are presented as the logarithm of the ratio of the amount of band $\mathrm{B}$ at a given chase time $(\mathrm{P})$ to the amount $\left(\mathrm{P}_{0}\right)$ at the beginning of the experiment. The turnover rate of immature form (band B) of wt-CFTR is shown as the slope of the line plotting the natural logarithm of the $\mathrm{P} / \mathrm{P}_{0}$ ratio against time. (C) Efficiency of conversion of the immature form (band $\mathrm{B}$ ) into the mature form (band C) of wt-CFTR in cells transfected with BAG-1. Conversion of band $B$ into band $C$ is shown as a percentage of the total mature protein recovered at a certain chase time relatively to the total amount of labelled immature protein (band $\mathrm{B}$ ) at the end of the pulse period $(\mathrm{t}=0$ ). Symbols and error bars are means \pm SEM of the values at each point. Squares and diamonds represent data from BAG-1 transfected cells and empty-vector control, respectively. Images are representative of a total of $\mathrm{n}=13$ experiments.

maturation efficiency of wt- CFTR in mock- and BAG-1-transfected cells (Fig. 2C) being of approximately $40 \%$, in agreement with previous studies in these cells $[5,16,17]$.

\section{In vivo effect of BAG-1 on the processing and degradation of F508del-CFTR}

Similarly to wt-CFTR experiments, we also assessed the effect of BAG-1 overexpression on the turnover and processing of F508del-CFTR stably expressed in CHO cells (Fig. 3A). Data show that the turnover rate of immature F508del-CFTR in cells transfected with in BAG-1 is decreased in comparison to that in cells transfected with the empty vector (Fig. 3B). Although small, this effect was reproducible and statistically significant $(\mathrm{p}<0.05)$. Nevertheless, we could not detect maturation of F508del-CFTR into the fully-glycosylated form under BAG-1 overexpression (Fig. 3A, lanes 7-12).

To further characterize the stabilization of F508del-CFTR by BAG-1, we determined whether inhibition of the proteasome further enhanced CFTR stability under BAG-1 overexpression. Summary of data from pulse-chase analysis (Fig. 3C) shows that the proteasome inhibitor ALLN causes a stabilization of immature F508del-CFTR in mocktransfected cells when compared to cells incubated only with DMSO (compare $1^{\text {st }}$ and $2^{\text {nd }}$ bars in Fig. 3C). As shown above, transfection with BAG-1 causes a stabilization of immature 
Fig. $\quad 3 . \quad B A G-1$ overexpression stabilizes the immature form of F508del-CFTR. (A) CHO cells stably expressing F508delCFTR were transfected either with the pcDNA3BAG-1 construct (lanes 7-12) or with the pcDNA3 empty vector (mock) (lanes 1-6) and pulse-chased followed by CFTR IP as described in Fig. 2. Images are representative of a total of $n=5$ experiments. (B) Turnover rate of immature form (band B) of F508del-CFTR in cells transfected with BAG1 or empty vector is graphically displayed as in Fig. 2B. Symbols and error bars are means \pm

A.

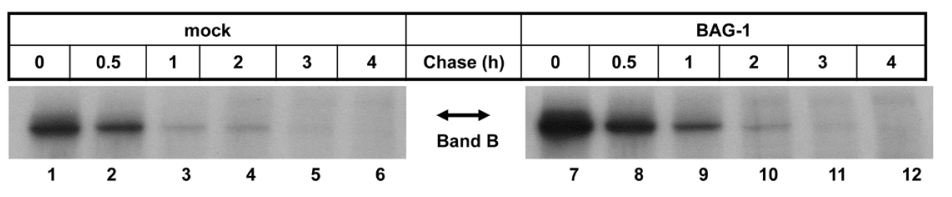

B. Band B turnover

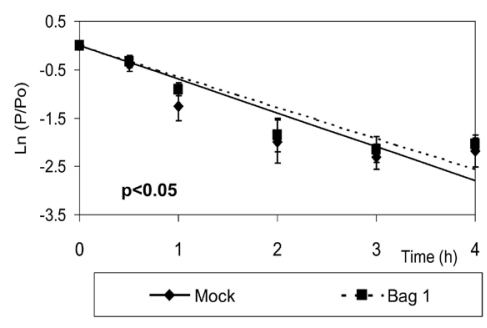

C.

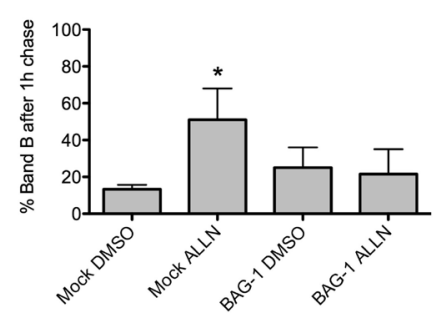

D.

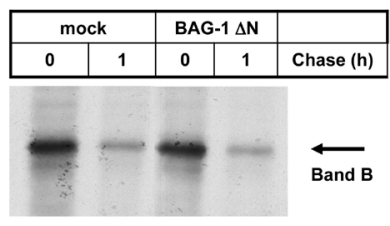

E.

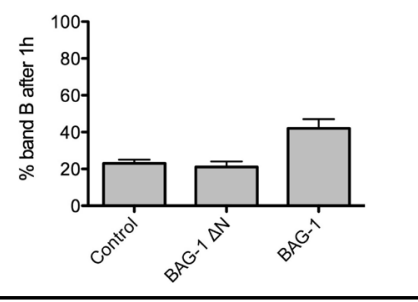

SEM of the values at each point. Differences were considered as significant for $p$ values $<0.05$. (C) Summary of data from experiments where CHO cells stably expressing F508del-CFTR and transfected with either the pEGFP-BAG-1 construct or the empty vector (mock), were incubated with ALLN or with vehicle alone (DMSO) and pulse-chased followed by CFTR IP $(n=3)$. Data are expressed as $\%( \pm$ SEM) of band B remaining after the $1 \mathrm{~h}$ chase. (D) CHO cells stably expressing F508del-CFTR were transfected with either the pcDNA3BAG-1- $\Delta \mathrm{N}$ construct or the pcDNA3 empty vector (mock) and pulse-chased followed by CFTR IP. Time points analysed were at $0 \mathrm{~h}$ and $1 \mathrm{~h}$ after pulse. Images are representative of a total of $n=3$ experiments. (E) Amount of Band B (shown as percentage of initial amount) remaining after the 1 h chase for cells expressing F508del-CFTR and transfected with BAG-1 full length, BAG-1- $\Delta \mathrm{N}$ or the empty vector.

F508del-CFTR (compare $1^{\text {st }}$ and $3^{\text {rd }}$ lanes in Fig. 3C). However, ALLN causes no further stabilization of immature F508del-CFTR in BAG-1-transfected cells in comparison to vehicletreated cells (Fig. 3C, last two bars), suggesting that the presence of BAG-1 may prevent F508del-CFTR from the UPP.

To further characterize this effect, we also assessed if this stabilization was observed if cells were transfected with BAG-1- $\Delta \mathrm{N}$, a BAG-1 variant lacking the N-terminus, corresponding to its ubiquitin-like domain (Ub-LD). F508del-CFTR Band B stability was assessed by pulsechase in cells transfected with either this variant or mock-transfected (Fig. 3D). Quantification of Band B remaining at $1 \mathrm{~h}$ of chase shows that, contrary to full-length BAG-1, this deletion variant does not promote stabilization of mutant CFTR (Fig. 3E), which presents turnover levels similar to those in the untransfected control.

Down-regulation of BAG-1 destabilizes F508del-CFTR expressed in human bronchial cells

In order to assess whether BAG-1 down-regulation caused the opposite effect on CFTR, we had to use a different cell line because neither CHO nor BHK cells express BAG1 endogenously. Accordingly, we used the human bronchial epithelial cell lines CFBE, transduced to stably express either wt- or F508del-CFTR. These cell lines have endogenous BAG-1 expression, thus allowing its down-regulation through siRNA transfection. 


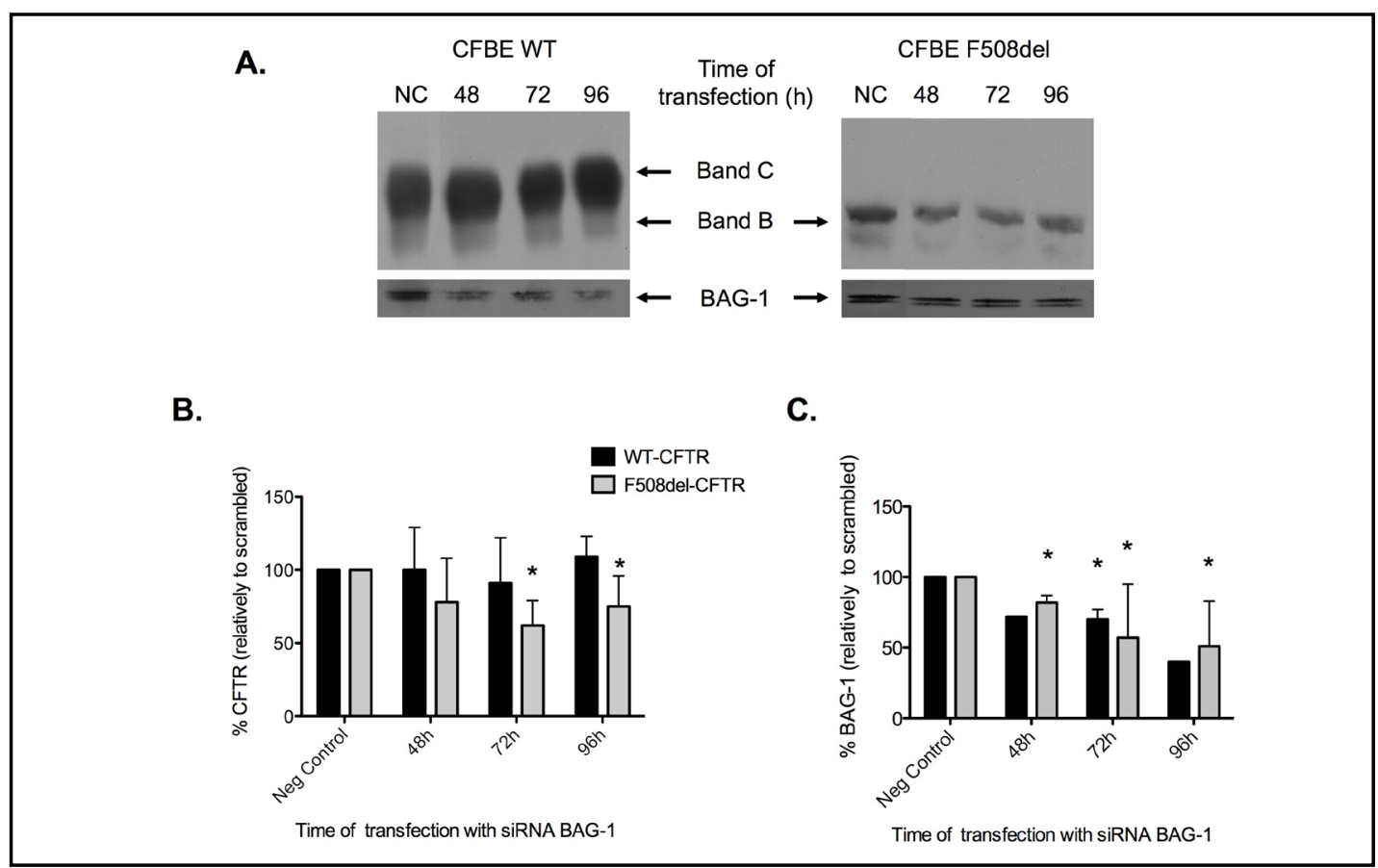

Fig. 4. Down-regulation of BAG-1 in human bronchial epithelial cells destabilizes F508del-CFTR. (A) CFBEwt or -F508del cells were transfected with either BAG-1 siRNA or a scrambled siRNA. 48h, $72 \mathrm{~h}$ or $96 \mathrm{~h}$ posttransfection, protein extracts were prepared and CFTR analysed by Western blot. (B) Levels of CFTR were determined by densitometry and plotted as the percentage of CFTR at steady state in BAG-1 transfected samples normalized to levels of CFTR at steady-state in cells transfected with scrambled siRNA. (C) Levels of BAG-1 were determined by densitometry and plotted as the percentage of CFTR at steady state in BAG-1 transfected samples normalized to levels of CFTR at steady-state in cells transfected with scrambled siRNA. Asterisk indicates statistically significant difference from the same cells transfected with scrambled siRNA $(\mathrm{p}<0.05)$.

CFBE-wt or F508del cells were transfected with either a siRNA specific for BAG-1 or a scrambled siRNA (negative control). Protein extracts were collected $48 \mathrm{~h}, 72 \mathrm{~h}$ or $96 \mathrm{~h}$ posttransfection and analysed by WB for either BAG-1 or CFTR (Fig. 4A). We were able to detect down-regulation of BAG-1 levels to about $70 \%$ of control-transfected cells (Fig. 4A-C). At this reduction of BAG-1 levels, we detected a decrease in the steady-state levels of F508del-CFTR that is, however, not observed for wt-CFTR (Fig. 4B, compare grey and black bars).

\section{Characterization of a possible association in vivo between of BAG-1 and CFTR}

Since BAG-1 has been shown to interact directly with multiple cellular targets, it seems plausible that BAG-1 may also associate in vivo with CFTR. However, since BAG-1 can also bind to Hsc70/Hsp70 [21, 22] and Hsc70/Hsp70 also associates with CFTR, an indirect interaction between CFTR and BAG-1 can also occur using Hsc70/Hsp70 as mediator.

To distinguish between these two possibilities, we used additional BAG-1 constructs, which also clarify which of the two BAG-1 functional domains (C-term-Hsp70-binding or $\mathrm{N}$-term-Ub-LD) are required for the in vivo CFTR/BAG-1 interaction. Constructs used were (Fig. 5A): BAG-1- $\Delta \mathrm{N}$ lacking the Ub-like domain (Ub-LD); BAG-1- $\Delta \mathrm{C}$ that misses the critical residues for Hsc70/Hsp70 interaction, also called the BAG domain (BD); and BAG-1 90-200, missing the first 90 aa (Ub-LD) and the last 19 aa (part of the BD), used here as a null control.

Results from pull-down of CFTR followed by immunoblotting of BAG-1 (Fig. 5B) show that full-length BAG-1 associates with both wt-CFTR (lane 2) and F508del-CFTR (lane 7), but in higher yields with the latter. The same result was obtained for BAG-1- $\Delta \mathrm{C}$, lacking the aa residues required for interaction with Hsc70/Hsp70, with both wt-CFTR and F508del-CFTR 
Fig. 5. BAG-1 associates directly with CFTR and decreases levels of ubiquitinated CFTR. (A) Schematic representation of BAG1 showing the different domains of the protein and the different constructs used in the present study. Numbers refer to amino acid residues. BAG-1- $\Delta \mathrm{N}$ construct has the ubiquitin (Ub)-like domain (Ub-LD) deleted, BAG-1 $\Delta \mathrm{C}$ construct lacks the aa residues required for interaction with Hsc70/Hsp70 and BAG-1 90-200 construct lacks both functional domains. (B) Pull-down of CFTR followed by immunoblotting of BAG-1. IP of CFTR, in baby hamster kidney (BHK) wt-CFTR or F508del-CFTR cells transfected with the BAG-1 constructs shown in (A) namely: full length BAG-1 (lanes 2 and 7); BAG-1 $1 \Delta \mathrm{N}$ (lanes 3 and 8); BAG-1 $\Delta \mathrm{C}$ (lanes 4 and 9); BAG-1 90-200 (lanes 5 and 10); or with the empty vector (lanes 1 and 6) as a control, was carried out as described in Methods. Proteins recovered in CFTR pulldowns were then separated by SDS-PAGE and immunoblotted for BAG-1 (position indicated by arrows). The band detected in the mock-transfected cells (lanes1 and 6), probably IgG, was discarded as unspecific. To assess for CFTR immunoprecipitation, an aliquot of the immunoprecipitate was also assessed for CFTR (middle panel). As a loading control aliquots of the total lysates were removed prior to the IP, separated by SDS-PAGE and immunoblotted for beta-actin (lower panel). (C) Pull-down of CFTR followed by immunoblotting of Ub under BAG-1 overexpression and ALLN-treatment. IP of CFTR, in CHO wt-CFTR and F508del-CFTR cells transfected with BAG-1 (lanes 1, 2 and 5,6) or the empty vector (lanes 3, 4 and 7, 8 ) and in the presence (lanes 2, 4, 6, 8) or in the absence (lanes 1, 3, 5, 7) of ALLN, was carried out as in Methods. Proteins recovered in CFTR pull-downs were then separated by SDS-PAGE and immunoblotted for Ub (upper panel). As a loading control aliquots of the total lysates were removed prior to the IP, separated by SDS-PAGE and immunoblotted for CFTR, beta-actin and BAG-1 (3 lower panels, respectively). Data show that ALLN does not cause an increase in the ubiquitinated forms of F508del-CFTR in cells overexpressing BAG-1 (lane 6) in contrast to the effect of this compound in mock-transfected cells (lane 8) or in wt-CFTR expressing cells either BAG-1 (lane 2) or mock-transfected (lane 4). (D) Pull-down of CFTR followed by immunoblotting of Ub under BAG-1 $\triangle \mathrm{N}$ overexpression and ALLN-treatment. IP of CFTR in F508del-CFTR CHO cells transfected with BAG- $1 \Delta \mathrm{N}$ (lanes 3 and 4) and in the presence (lanes 2, 4) or in the absence (lanes 1,3 ) of ALLN was carried out as described in (C). Loading controls as in (C).

(Fig. 5B, lanes 4 and 9, respectively). These results demonstrate that BAG-1 interacts with CFTR independently of Hsc-70. In contrast, BAG-1 deletion mutants, BAG-1- $\triangle \mathrm{N}$ and BAG-1 90-200 were not found to associate with either wt- (lanes 3 and 5) or F508del-CFTR (lanes 8 and 10), thus demonstrating that the Ub-LD is required for the BAG-1/CFTR interaction. The upper band detected in the mock-transfected cells (lanes1 and 6) is unspecific, probably IgG. 
Together with the stabilization effect that it causes on F508del-CFTR (Fig. 3), these results suggest that, through direct binding via its Ub-LD, BAG-1 may compete with Ub towards F508del-CFTR binding, partially preventing proteasomal degradation of the latter.

To further investigate this mechanistic role of BAG-1 as a competitor of ubiquitin for F508del-CFTR binding, we examined the appearance of ubiquitinated forms of CFTR under BAG-1-overexpression. To this end, CHO cells stably expressing wt- or F508del-CFTR and transfected with either BAG-1 or the empty vectors were incubated with the proteasome inhbitor ALLN or DMSO (vehicle). After CFTR IP, its ubiquitinated forms were detected by WB with an anti-polyUb Ab (Fig. 5C). Results show that treatment of wt-CFTR expressing cells with ALLN causes the appearance of ubiquitinated (high molecular mass) forms of CFTR in either mock- or BAG-1-transfected cells in equivalent amounts (Fig. 5C, lanes 2 and 4 , respectively). Similarly, when mock-transfected F508del-CFTR cells are treated with ALLN, a significant accumulation of Ub-CFTR is observed in comparison to vehicle-treated cells (Fig. 5C, lanes 8 and 7, respectively). In contrast, in BAG-1-transfected cells, ALLN treatment does not cause Ub-CFTR to accumulate, as shown by the similar (and low) levels of Ub-CFTR forms in F508del-CFTR cells treated with either ALLN or vehicle (Fig. 5C, lanes 6 and 5, respectively). These data further indicate that BAG-1 overexpression precludes F508delCFTR from undergoing polyubiquitination.

Moreover, when the same experiment was performed using BAG-1- $\Delta \mathrm{N}$ (lacking the Ub-LD) instead of full-length BAG-1, to transfect F508del-CFTR expressing cells, the ALLNtreatment caused again appearance of high molecular Ub-F508del-CFTR forms, which were in equivalent amounts to those in mock-transfected cells (Fig. 5D, lanes 4 and 2, respectively). This result shows that the Ub-LD of BAG-1, above demonstrated to be required for the association of BAG-1 with CFTR, is required to prevent occurrence of F508del-CFTR ubiquitination under ALLN.

\section{Discussion}

The main purpose of this study was to contribute to the basic understanding of the mechanism of ER retention and ER-associated degradation (ERAD) of the most common mutant protein responsible for Cystic Fibrosis - F508del-CFTR. The identification of components of the machinery retaining the mutant protein in the ER and selecting it for the proteolytic pathway may lead to a better understanding of this process. Ultimately, this may help to develop agents that promote cell surface rescuing of F508del-CFTR, in a therapeutic strategy aimed at correcting the basic defect in CF.

Overexpression of BAG-1 confers protection from the stress response

Induction of cytosolic molecular chaperones, e.g., Hsp70 and Hsp90, is a major protective mechanism against cellular stress and constitutes the so-called HS response. The activation of this pathway occurs mostly through transcriptional regulation of heat shock factor 1 (HSF1), which binds to HS elements located in the promoter region of HS genes leading to an increase in their expression. The response to HS is also subjected to negative feedback regulation through which overproduction of Hsp70 attenuates HSF1 activity by direct binding to the monomeric factor, inactivating it (reviewed in [35]).

Here, we show that the well-known induction of Hsp70, which follows a HS treatment as widely described $[36,37]$, is drastically reduced in cells overexpressing BAG-1. We interpret these data by the described effect of BAG-1 in promoting substrate release from Hsp70 [21, 22], which thus makes more Hsp70 available for the feedback inhibition of HSF1 [35]. This constitutes novel in vivo evidence of an inhibitory effect of BAG-1 on Hsp70 induction by HS. Indeed, BAG-1 has been described to induce conformational changes in Hsp70 upon binding to its ATPase domain [38], this interaction plausibly reduces Hsp70 binding affinity to ATP, hence inhibiting the Hsp70 chaperone activity [39]. This is consistent with the previously reported role of BAG-1 inhibiting Hsp70 activity in vivo, even overriding the stimulatory 
action of another Hsp70 co-chaperone, Hip [40]. This inhibitory effect of BAG-1 in vivo is compatible with a role for BAG-1 in recovery from stress acting as a "buffering protein" helping to restore the levels of induced chaperones back to resting conditions.

Interestingly, we also found here the opposite effect, i.e., that the expression levels of BAG-1 detected after BAG-1 transfection are consistently reduced in cells subjected to HS, suggesting that this effect may result from the high levels of chaperones induced by HS. Alternatively, this may be simply due to the overall protein synthesis which occurs during HS due to preference given to 5'Cap-independent mRNAs translation [34, 41]. Notwithstanding, it was shown that BAG-1 itself is a substrate of the E3-Ub ligase, CHIP and ubiquitin attachment to BAG-1 does not interfere with Hsc/Hsp70 binding [24, 42]. Hence the high Hsp70 levels induced by HS may promote formation of the CHIP/BAG-1/Hsp70 ternary complex targeting more BAG-1 for degradation.

$B A G$-1 associates in vivo with CFTR independently of Hsc70 and stabilizes the immature form of F508del CFTR

Results shown here demonstrate that BAG-1 associates in vivo both with wt-CFTR and F508del-CFTR, but in higher yields with the latter (Fig. 5). Data also reveal that this interaction is independent of Hsc70, as BAG-1 lacking the HSc70/Hsp70 interaction domain still interacts with CFTR (wt- or F508del) $[3,5]$. Several reports in the literature have shown direct association of BAG-1 with different proteins namely Raf-1 kinase [30]; retinoic acid receptor [43]; ubiquitin [44]; hepatocyte growth factor receptor and platelet-derived growth factor [45].

However, because Hsp70 was also found in co-IPs of CFTR and BAG-1, we cannot exclude that the role of BAG-1 on F508del-CFTR stabilization is completely independent of the Hsc70 chaperone. Moreover, since BAG-1 deletion mutants without the Ub-LD were not found in association with CFTR (either wt or F508del), we conclude that the Ub-like region is required for the direct interaction between BAG-1 and CFTR.

Our data shown here demonstrate that the immature form of F508del-CFTR is significantly stabilized under BAG-1 overexpression. However that effect is abolished when the Ub-LD is missing. Moreover, we have also demonstrated that in human bronchial cells the down-regulation of endogenous BAG-1 destabilizes F508del-CFTR, but not wt-CFTR.

Notwithstanding, BAG-1 overexpression does not cause F508del-CFTR to undergo maturation, indicating that the stabilization occurs either for an off-pathway intermediate (not occurring for wt-CFTR) or at a checkpoint where wt-CFTR is not halted [16]. Although previous studies suggested that BAG-1 stimulates sorting of client proteins to the proteasome [24], this effect was shown to involve Hsp70 (see above). Our data shown here clearly demonstrate that the stabilizing effect exerted by BAG-1 on F508del-CFTR is independent of its Hsp70 binding domain. It thus seems plausible that the effect we observe here for BAG-1, which is mediated by the Ub-like domain overrides its role in UPP described previously [24]. Notwithstanding, it remains possible that the F508del-CFTR stabilization by BAG-1 is due to its release from Hsc70/Hsp70 machinery, which has been proposed to be involved in the major checkpoint for F508del-CFTR degradation [16].

On the other hand, the occurrence of BAG-1/CFTR complexes containing Hsp70 (also detected here) could still promote F508del-CFTR degradation, in accordance with the role for BAG-1 in stimulating the CHIP-mediated degradation, through formation of the Hsc70/ BAG-1/CHIP complex [24]. However, the direct association of BAG-1 to CFTR, competing ubiquitin out of F508del-CFTR binding, seems to override the former, but just for F508delCFTR and not for wt-CFTR where the two opposing roles seem to balance each other.

Similarly to our observations for F508del-CFTR, overexpression of BAG-1 was also shown to induce an increase in levels of tau protein, which was shown to be due to an inhibition of protein degradation by the proteasome together with upregulation of tau refolding by Hsp70 [46]. In fact, BAG-1 cannot be envisaged as a general accelerator of protein degradation, as different target proteins have been shown to be differently affected by the regulatory function of BAG-1: while it enhances the function of Bcl-2, Raf-1 protein 
kinase [18], androgen receptor and hepatocyte growth factor receptor [47], it inhibits the retinoic acid receptor and glucocorticoid receptor activities [43].

\section{BAG-1 competes with ubiquitin for F508del-CFTR binding}

Further characterization of F508del-CFTR stabilization by BAG-1, showed that treatment of BAG-1-transfected cells with the proteasome inhibitor ALLN caused no additional stabilization of F508del-CFTR, in deep contrast to mock-transfected cells. Moreover, in BAG-1-overexpressing cells, a significant decrease in ubiquitinated forms of F508del-CFTR was observed in comparison to mock-transfected cells. Of note, the Ub-LD of BAG-1 is required to prevent polyubiquitination of F508del-CFTR under ALLN. Overall, these data are compatible with a mechanism in which BAG-1 stabilizes F508del-CFTR by direct binding (see above), most probably by competing out ubiquitin and partially avoiding F508del-CFTR from undergoing proteasomal degradation.

Altogether, our data are still compatible with a twofold role of BAG-1 on F508del-CFTR stabilization also involving the Hsc70 chaperone: firstly by promoting release of F508delCFTR from the Hsc70/Hsp70 machinery that targets the mutant to degradation via CHIP interaction [48] and secondly by competing with ubiquitin through direct binding of F508del-CFTR, thus preventing its proteasomal proteolysis.

\section{Abbreviations}

$\mathrm{Ab}$ (antibody); $\mathrm{ABC}$ (ATP-binding cassette); BAG (Bcl-2-associated athanogenes); BHK cells (baby hamster kidney cells); CF (cystic fibrosis); CFTR (cystic fibrosis transmembrane conductance regulator); CHO cells (Chinese hamster ovary cells); ER (endoplasmic reticulum); ERAD (ER associated degradation); ERQC (endoplasmic reticulum quality control); HS (heat shock); IP (immunoprecipitation); NBD (nucleotide binding domain); Ub (ubiquitin); Ub-LD (Ub-like domain); UPP (ubiquitin-proteasome pathway); wt (wild type).

\section{Acknowledgements}

This work was supported by strategic grants PEst-OE/BIA/UI4046/2011 FCT, Portugal to BioFIG, FCT/MCTES grants PTDC/SAU-GMG/122299/2010 and PTDC/BIABCM/112635/2009, a grant from Fundação Calouste Gulbenkian (Portugal), and postdoctoral fellowships SFRH/BPD/1905/2004 (FM) and Praxis XXI BPD/18811/98 (IV) from FCT, Portugal. We thank Prof R Morimoto and J Song (Northwestern University, Evanston, IL, USA) for the BAG-1 constructs, Prof JR Riordan (UNC, Chapel Hill, NC, USA) for the CFTR-CHO cell lines and Prof. WB Guggino (Johns Hopkins University School of Medicine, Baltimore, MD, USA) for the 169 anti-CFTR Ab.

\section{References}

1 Welsh M, Tsui LC, Boat TF, Beaudet AL: Cystic fibrosis; in Scriver CR, Beaudet AL, Sly WS, Valle D (eds): The metabolic and molecular basis of inherited disease. New York, McGraw-Hill, Inc., 1995, vol 7, pp 37993876.

-2 Amaral MD: Cftr and chaperones: Processing and degradation. J MolNeurosci 2004;23:41-48.

3 Yang Y, Janich S, Cohn JA, Wilson JM: The common variant of cystic fibrosis transmembrane conductance regulator is recognized by hsp70 and degraded in a pre-golgi nonlysosomal compartment. Proc Natl Acad Sci USA 1993;90:9480-9484. 
Pind S, Riordan JR, Williams DB: Participation of the endoplasmic reticulum chaperone calnexin (p88, ip90) in the biogenesis of the cystic fibrosis transmembrane conductance regulator. JBiolChem 1994;269:1278412788.

5 Farinha CM, Nogueira P, Mendes F, Penque D, Amaral MD: The human dnaj homologue (hdj)-1/heat-shock protein (hsp) 40 co-chaperone is required for the in vivo stabilization of the cystic fibrosis transmembrane conductance regulator by hsp70. BiochemJ 2002;366:797-806.

6 Meacham GC, Lu Z, King S, Sorscher E, Tousson A, Cyr DM: The hdj-2/hsc70 chaperone pair facilitates early steps in cftr biogenesis. EMBO J 1999;18:1492-1505.

7 Loo MA, Jensen TJ, Cui L, Hou Y, Chang XB, Riordan JR: Perturbation of hsp90 interaction with nascent cftr prevents its maturation and accelerates its degradation by the proteasome. EMBO J 1998;17:6879-6887.

-8 Younger JM, Ren HY, Chen L, Fan CY, Fields A, Patterson C, Cyr DM: A foldable cftr\{delta\}f508 biogenic intermediate accumulates upon inhibition of the hsc70-chip e3 ubiquitin ligase. J Cell Biol 2004;167:10751085.

-9 Alberti S, Bohse K, Arndt V, Schmitz A, Hohfeld J: The cochaperone hspbp1 inhibits the chip ubiquitin ligase and stimulates the maturation of the cystic fibrosis transmembrane conductance regulator. MolBiolCell 2004;15:4003-4010.

10 Arndt V, Daniel C, Nastainczyk W, Alberti S, Hohfeld J: Bag-2 acts as an inhibitor of the chaperoneassociated ubiquitin ligase chip. Mol Biol Cell 2005;16:5891-5900.

11 Schmidt BZ, Watts RJ, Aridor M, Frizzell RA: Cysteine string protein promotes proteasomal degradation of the cystic fibrosis transmembrane conductance regulator (cftr) by increasing its interaction with the c terminus of hsp70-interacting protein and promoting cftr ubiquitylation. J Biol Chem 2009;284:41684178.

12 Cheng SH, Gregory RJ, Marshall J, Paul S, Souza DW, White GA, O’Riordan CR, Smith AE: Defective intracellular transport and processing of cftr is the molecular basis of most cystic fibrosis. Cell 1990;63:827-834.

13 Lukacs GL, Mohamed A, Kartner N, Chang XB, Riordan JR, Grinstein S: Conformational maturation of cftr but not its mutant counterpart (delta f508) occurs in the endoplasmic reticulum and requires atp. EMBO J 1994;13:6076-6086.

14 Jensen TJ, Loo MA, Pind S, Williams DB, Goldberg AL, Riordan JR: Multiple proteolytic systems, including the proteasome, contribute to cftr processing. Cell 1995;83:129-135.

15 Ward CL, Omura S, Kopito RR: Degradation of cftr by the ubiquitin-proteasome pathway. Cell 1995;83:121127.

16 Farinha CM, Amaral MD: Most f508del-cftr is targeted to degradation at an early folding checkpoint and independently of calnexin. MolCell Biol 2005;25:5242-5252.

17 Roxo-Rosa M, Xu Z, Schmidt A, Neto M, Cai Z, Soares CM, Sheppard DN, Amaral MD: Revertant mutants g550e and 4rk rescue cystic fibrosis mutants in the first nucleotide-binding domain of cftr by different mechanisms. ProcNatlAcadSciUSA 2006;103:17891-17896.

18 Takayama S, Sato T, Krajewski S, Kochel K, Irie S, Millan JA, Reed JC: Cloning and functional analysis of bag1: A novel bcl-2-binding protein with anti-cell death activity. Cell 1995;80:279-284.

19 Kabbage M, Dickman MB: The bag proteins: A ubiquitous family of chaperone regulators. Cell Mol Life Sci 2008;65:1390-1402.

20 Wang HG, Takayama S, Rapp UR, Reed JC: Bcl-2 interacting protein, bag-1, binds to and activates the kinase raf- 1. Proc Natl Acad Sci U S A 1996;93:7063-7068.

21 Hohfeld J, Jentsch S: Grpe-like regulation of the hsc70 chaperone by the anti- apoptotic protein bag-1 [published erratum appears in embo j 1998 feb 2;17:847]. EMBO J 1997;16:6209-6216.

-22 Takayama S, Bimston DN, Matsuzawa S, Freeman BC, Aime-Sempe C, Xie Z, Morimoto RI, Reed JC: Bag-1 modulates the chaperone activity of hsp70/hsc70. EMBO J 1997;16:4887-4896.

23 Luders J, Demand J, Hohfeld J: The ubiquitin-related bag-1 provides a link between the molecular chaperones hsc70/hsp70 and the proteasome. J BiolChem 2000;275:4613-4617.

24 Alberti S, Demand J, Esser C, Emmerich N, Schild H, Hohfeld J: Ubiquitylation of bag-1 suggests a novel regulatory mechanism during the sorting of chaperone substrates to the proteasome. J BiolChem 2002;277:45920-45927.

25 Matsumura Y, David LL, Skach WR: Role of hsc70 binding cycle in cftr folding and endoplasmic reticulumassociated degradation. Mol Biol Cell 2011;22:2797-2809. 


\section{Cellular Physiology $\quad$ Cell Physiol Biochem 2012;30:1120-1133 and Biochemistry

-26 Takayama S, Reed JC: Molecular chaperone targeting and regulation by bag family proteins. NatCell Biol 2001;3:E237-E241.

-27 Zhang F, Kartner N, Lukacs GL: Limited proteolysis as a probe for arrested conformational maturation of delta f508 cftr [see comments]. Nat Struct Biol 1998;5:180-183.

28 Bebok Z, Mazzochi C, King SA, Hong JS, Sorscher EJ: The mechanism underlying cystic fibrosis transmembrane conductance regulator transport from the endoplasmic reticulum to the proteasome includes sec61 beta and a cytosolic, deglycosylated intermediary. J Biol Chem 1998;273:29873-29878.

- 29 Brimmell M, Burns JS, Munson P, McDonald L, O’Hare MJ, Lakhani SR, Packham G: High level expression of differentially localized bag- 1 isoforms in some oestrogen receptor-positive human breast cancers. $\mathrm{Br}$ J Cancer 1999;81:1042-1051.

-30 Song J, Takeda M, Morimoto RI: Bag1-hsp70 mediates a physiological stress signalling pathway that regulates raf-1/erk and cell growth. NatCell Biol 2001;3:276-282.

- 31 Crawford I, Maloney PC, Zeitlin PL, Guggino WB, Hyde SC, Turley H, Gatter KC, Harris A, Higgins CF: Immunocytochemical localization of the cystic fibrosis gene product cftr. Proc Natl Acad Sci USA 1991;88:9262-9266.

-32 Younger JM, Chen L, Ren HY, Rosser MF, Turnbull EL, Fan CY, Patterson C, Cyr DM: Sequential qualitycontrol checkpoints triage misfolded cystic fibrosis transmembrane conductance regulator. Cell 2006;126:571-582.

-33 Zhang Y, Nijbroek G, Sullivan ML, McCracken AA, Watkins SC, Michaelis S, Brodsky JL: Hsp70 molecular chaperone facilitates endoplasmic reticulum-associated protein degradation of cystic fibrosis transmembrane conductance regulator in yeast. Mol Biol Cell 2001;12:1303-1314.

- 34 Theodorakis NG, Morimoto RI: Posttranscriptional regulation of hsp70 expression in human cells: Effects of heat shock, inhibition of protein synthesis, and adenovirus infection on translation and mrna stability. Mol Cell Biol 1987;7:4357-4368.

-35 Morimoto RI: Regulation of the heat shock transcriptional response: Cross talk between a family of heat shock factors, molecular chaperones, and negative regulators. Genes Dev 1998;12:3788-3796.

-36 Burdon RH, Slater A, McMahon M, Cato AC: Hyperthermia and the heat-shock proteins of hela cells. Br] Cancer 1982;45:953-963.

-37 Li GC, Werb Z: Correlation between synthesis of heat shock proteins and development of thermotolerance in chinese hamster fibroblasts. Proc Natl Acad Sci USA 1982;79:3218-3222.

- 38 Brive L, Takayama S, Briknarova K, Homma S, Ishida SK, Reed JC, Ely KR: The carboxyl-terminal lobe of hsc70 atpase domain is sufficient for binding to bag1. BiochemBiophysResCommun 2001;289:1099-1105

-39 Sondermann H, Scheufler C, Schneider C, Hohfeld J, Hartl FU, Moarefi I: Structure of a bag/hsc70 complex: Convergent functional evolution of hsp70 nucleotide exchange factors. Science 2001;291:1553-1557.

-40 Nollen EA, Kabakov AE, Brunsting JF, Kanon B, Hohfeld J, Kampinga HH: Modulation of in vivo hsp70 chaperone activity by hip and bag-1. J Biol Chem 2001;276:4677-4682.

-41 Duncan R, Hershey JW: Heat shock-induced translational alterations in hela cells. Initiation factor modifications and the inhibition of translation. J Biol Chem 1984;259:11882-11889.

42 Demand J, Alberti S, Patterson C, Hohfeld J: Cooperation of a ubiquitin domain protein and an e3 ubiquitin ligase during chaperone/proteasome coupling. CurrBiol 2001;11:1569-1577.

43 Liu R, Takayama S, Zheng Y, Froesch B, Chen GQ Zhang X, Reed JC, Zhang XK: Interaction of bag- 1 with retinoic acid receptor and its inhibition of retinoic acid-induced apoptosis in cancer cells. JBiolChem 1998;273:16985-16992.

-44 Matsuzawa S, Takayama S, Froesch BA, Zapata JM, Reed JC: P53-inducible human homologue of drosophila seven in absentia (siah) inhibits cell growth: Suppression by bag-1. EMBO J 1998;17:2736-2747.

45 Bardelli A, Longati P, Albero D, Goruppi S, Schneider C, Ponzetto C, Comoglio PM: Hgf receptor associates with the anti-apoptotic protein bag-1 and prevents cell death. EMBO J 1996;15:6205-6212.

-46 Elliott E, Tsvetkov P, Ginzburg I: Bag-1 associates with hsc70/tau complex and regulates the proteasomal degradation of tau protein. J Biol Chem 2007;282:37276-37284.

47 Froesch BA, Takayama S, Reed JC: Bag-1l protein enhances androgen receptor function. JBiolChem 1998;273:11660-11666.

48 Meacham GC, Patterson C, Zhang W, Younger JM, Cyr DM: The hsc70 co-chaperone chip targets immature -cftr for proteasomal degradation. NatCell Biol 2001;3:100-105. 